\title{
Angiographical Changes Induced by Tiny Coronary Fistula
}

pISSN 2508-707X / elSSN 2508-7088 https://doi.org/10.22468/cvia.2017.00059 CVIA 2017;1(3):208-209

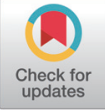

\author{
Tatsuya Saigusa', Hitoshi Matsuo ${ }^{2}$, Takahiko Suzuki ${ }^{3}$ \\ 'Department of Cardiovascular Medicine, Shinshu University Hospital, Matsumoto, Japan \\ ${ }^{2}$ Department of Cardiovascular Medicine, Gifu Heart Center, Gifu, Japan \\ ${ }^{3}$ Department of Cardiovascular Medicine, Toyohashi Heart Center, Toyohashi, Japan
}

Received: March 26, 2017

Revised: June 30, 2017

Accepted: July 6, 2017

\section{Corresponding author}

Tatsuya Saigusa, MD

Department of Cardiovascular Medicine, Shinshu University Hospital, 3-1-1 Asahi,

Matsumoto 390-8621, Japan

Tel: 81-263-37-3352

Fax: 81-263-37-2573

E-mail: tatsuwan@yahoo.co.jp

\begin{abstract}
An 82-year-old female presented with angina pectoris due to mid-right coronary artery (RCA) stenosis. Percutaneous coronary intervention ( $\mathrm{PCI}$ ) was performed with optical coherence tomography (OCT). When OCT was performed with manual coronary flushing to obtain a clearer image, the contrast media seemed to extravasate from the coronary artery, resembling a coronary perforation; however, the patient did not have any chest pain and her vital signs were stable. Coronary angiography (CAG) performed immediately after the finding showed no abnormalities and the final CAG did not show any abnormalities. Coronary computed tomography angiography after $\mathrm{PCI}$ revealed a micro-fistula from the RCA to the right ventricle. With normal injection pressure, contrast did not show the tiny coronary fistula, but it was detectable with high pressure. Our report represents an abrupt angiographical change due to a tiny coronary fistula.
\end{abstract}

Key words Coronary fistula · Percutaneous coronary intervention · High-pressure injection Coronary computed tomography angiography.

\section{INTRODUCTION}

A coronary fistula is an abnormal connection between the coronary artery and another vessel or cardiac chamber and is occasionally identified during coronary angiography (CAG). Although a coronary fistula can be visualized during CAG, in this case, after the angiography, the coronary fistula could not be detected. Coronary computed tomography angiography (CCTA) after percutaneous coronary intervention (PCI) revealed a micro-fistula from the RCA to the right ventricle. Our report represents an abrupt angiographical change due to a tiny coronary fistula which can be detected by CCTA.

\section{CASE REPORT}

An 82-year-old female patient was scheduled for CAG as a follow-up for a previous coronary artery bypass $(\mathrm{CAB})$ and coronary stent implantation that were performed without postoperative complications. She had undergone the CAB, which involved the left internal thoracic artery to mid-left descending artery and radial artery to left circumflex artery, because of an

(c) This is an Open Access article distributed under the terms of the Creative Commons Attribution Non-Commercial License (http://creativecommons.org/licenses/bync/4.0) which permits unrestricted non-commercial use, distribution, and reproduction in any medium, provided the original work is properly cited. angina of effort in 1998. New-onset chest discomfort at rest occurred in 2013 and total occlusion at the mid-right coronary artery (RCA) was detected, followed by the implantation of an everolimus-eluting stent (Xience-Xpedition ${ }^{\circledR} 3.25 / 38 \mathrm{~mm}$; Abbott Vascular, Chicago, IL, USA).

CAG at 9 months after stenting revealed a new coronary instent stenosis at the mid-RCA with significant myocardial ischemia, followed by PCI.

Frequency-domain optical coherence tomography (FD-OCT) was performed with manual rapid contrast flushing $(10 \mathrm{~mL} /$ $\mathrm{sec}$ ) after the coronary wire was passed through the stenosis to determine lumen size and tissue characteristics. The angiography at OCT resembled an oozing rupture of the coronary vessel (Supplementary Fig. 1A and Supplementary Movie 1 in the online-only Data Supplement); however, the patient was asymptomatic and her vital signs were generally stable. Echocardiography was performed to determine if cardiac tamponade had been caused by the coronary perforation, but no cardiac effusion was identified. CAG was repeated immediately, with a normal speed injection $(5 \mathrm{~mL} / \mathrm{sec})$, but the repeat CAG did not show any abnormalities (Supplementary Fig. 1B and Supplementary Movie 1 in the online-only Data Supplement).

FD-OCT showed partial dissection of the coronary vessel, but no signs of vessel perforation, therefore, we decided to con- 
tinue with the PCI. Pre-dilatation was performed with a scoring balloon, followed by treatment with a drug-coated balloon (SeQuent Please ${ }^{\circledR}$ 3.5/30 mm; B. Braun Melsungen AG, Berlin, Germany).

Echocardiography was repeated after the procedure, however, pericardial effusion was not detected. CCTA revealed some micro-coronary fistulas connecting the RCA to the right ventricular (RV) chamber (Supplementary Fig. 2) in the online-only Data Supplement); these were thought to be the cause of the angiographical finding.

\section{DISCUSSION}

This paper reports a micro-coronary fistula from the RCA to the RV that was revealed only when CAG with high-pressure injection was performed. Fistulas are occasionally encountered during CAG; however, they are usually large and visible. The majority of coronary fistulas are congenital and some coronary fistulas are acquired after infection, trauma, and iatrogenic injury, however, many minute fistulas can remain undetectable [1]. Based on this patient's previous history of CAB graft surgery, it is possible that this coronary fistula was a result of that procedure. Coronary fistulas are typically associated with CCTA, although in this case the lumen was relatively long and smooth. Iatrogenic coronary fistulas have been reported to show relatively short routes with an irregular lumen [2], therefore, we speculated that this was a congenital tiny fistula.

This case was characterized by abrupt angiographical change, even though the change did not occur in cases with typical coronary fistulas. This case is unique because the angiographical findings changed immediately after previous imaging. We speculated that the cause for these abrupt changes was attributable to the difference in the amount of contrast through the coronary fistula to the right ventricle. A typical coronary fistula is relatively large in diameter, therefore, a large amount of contrast through the fistula could visualize the route. For cases with tiny fistulas, a small amount of contrast is insufficient for visualizing the route. Interestingly, in this case, the contrast draining into the right ventricle through the tiny fistula could only be visualized with a high pressure injection.

This case showed that CCTA was useful for detecting the origin of the micro-coronary fistula, which connected the RCA to the RV. Many coronary fistulas are small and are accidentally detected with CAG [3]. For children, these fistulas are monitored because they can increase in size as the children grow and because increased flow may occur even for asymptomatic fistulas. Micro-fistulas, similar to this case, could be the pre-phase of a large fistula that could be seen on CAG. Larger coronary fistulas may lead to angina pectoris because of the coronary steal phenomenon that necessitates surgical repair. Tuncer et al. [4] revealed that micro-coronary fistulas were detected via angiography, but they could not demonstrate the fistula drainage location into because additional imaging modalities, such as CCTA or transesophageal echocardiography, were not performed. Based on these results, CCTA may reveal an immature fistula undetected by routine CAG and may be a more sensitive modality than CAG for follow-up.

Coronary fistulas have increased as children age [3], and if these large fistulas remain untreated, angina or heart failure could occur in $63 \%$ of these patients during adulthood [5]. In this case, fractional flow reserve showed no RCA ischemia after PCI. The reason that ischemia was not identified was attributable to adequate dilatation of the RCA lesion and less steal flow draining through the coronary fistula into the RV. Several microvessels maintain microcirculation in the myocardium but they are rarely revealed in clinical examination. In conclusion, asymptomatic fistulas should be monitored because of the possibility of fistula enlargement, and CCTA is a useful approach for identifying these small fistulas.

\section{Supplementary Movie Legends}

Movie 1. Coronary angiography with high-pressure injection during optical coherence tomography. Contrast medium, which is likely outside the coronary vessels, can be seen. Coronary angiography with normal injection shows no abnormal findings, immediately after the previous finding.

\section{Supplementary Materials}

The online-only Data Supplement is available with this article at https://doi. org/10.22468/cvia.2017.00059.

\section{Conflicts of Interest}

The authors declare that they have no conflict of interest.

\section{REFERENCES}

1. Latson LA. Coronary artery fistulas: how to manage them. Catheter Cardiovasc Interv 2007;70:110-116.

2. Schanzenbächer P, Bauersachs J. Acquired right coronary artery fistula draining to the right ventricle: angiographic documentation of first appearance following reperfusion after acute myocardial infarction, with subsequent spontaneous closure. Heart 2003;89:e22.

3. Mangukia CV. Coronary artery fistula. Ann Thorac Surg 2012;93:20842092.

4. Tuncer C, Eryonucu B, Batyraliev T, Gökce M, Yllmaz R, Akkoyun M, et al. Angiographic characteristics of coronary artery fistulas. Turk Kardiyol Dern Ars 2014;42:456-460.

5. Mavroudis C, Backer CL, Rocchini AP, Muster AJ, Gevitz M. Coronary artery fistulas in infants and children: a surgical review and discussion of coil embolization. Ann Thorac Surg 1997;63:1235-1242. 